DOI: 10.24867/MD.9.2017.2.69-72

Research paper

\title{
METHODOLOGY OF KNEE BONES MODELS 3D PRINTING BASED ON CT SERIES OF IMAGES
}

\author{
Zoran MILOJEVIĆ'1, * Slobodan TABAKOVIĆ1 - Milan ZELJKOVIĆ ${ }^{1}$ - Aleksandar ŽIVKOVIĆ1 - Slobodan NAVALUŠIĆ1 \\ 1 University of Novi Sad, Faculty of Technical Sciences, Novi Sad, Serbia
}

\author{
Received (26.01.2017); Revised (16.03.2017); Accepted (20.03.2017)
}

\begin{abstract}
In this paper a methodology for knee bones models 3D printing from DICOM series of images is presented. It would be very useful for doctors to see real models of knee bones and ligament attachment sites onto both bones before the ACL reconstruction. With physical bone model, orientation and position of operative equipment (tibial and femoral guides) could be planned before the operation. This could lead to more precise ACL reconstruction. In the introductory part of this paper ACL reconstruction method and some problems that may occur are presented. After that, model of procedure for knee bones $3 D$ printing is shown. First, a procedure for $3 D$ knee bone models generation is shown. For this purpose a program system is developed based on the VTK library. Generation of femoral transverse axis which enables tibial bone to rotate about femoral bone is very important. This axis enables knee flexion and extension and in ACL reconstruction, doctors rotate tibial bone about femoral bone which is fixed. Point clouds of femoral bone condyles are used for calculation of centres of best fit spheres of both condyles by least squares method. After that bone models are prepared for 3D printing. Because of the MakerBot Replicator $2 X$ printer limitations, model of the knee is printed in two parts which are then glued together.
\end{abstract}

Key words: DICOM, CT, 3D printing, ACL reconstruction, least squares method

\section{INTRODUCTION}

Most common surgically treated knee ligament is ACL (Anterior Cruciate Ligament) [1]. Its function is to prevent front movement of tibial bone in respect to femoral bone. Also, it provides lateral and rotational knee stability. Most often, ACL injury happens during sport activities. In Figure 1a, tibial and femoral bones and ACL are shown.

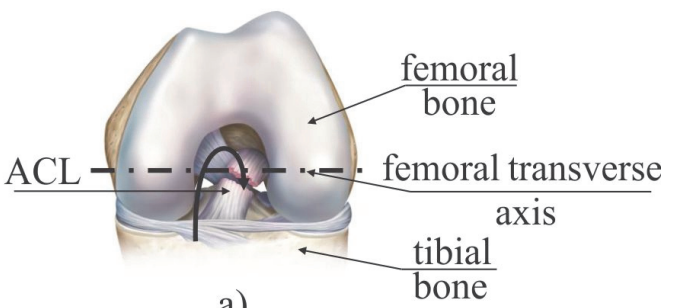

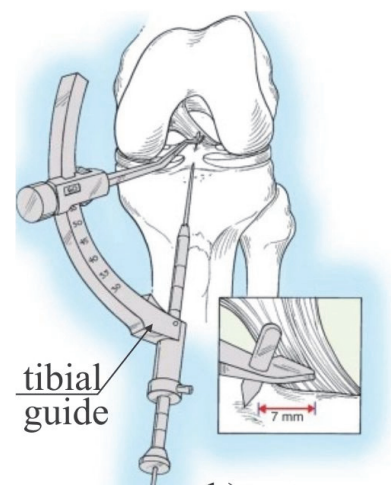

b)

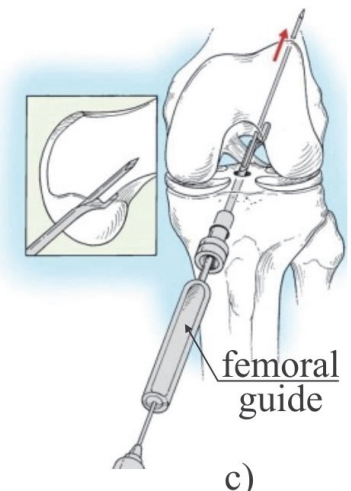

c)
Fig.1. a) Tibial and femoral bones and ACL, b) Tibial guide, and c) Femoral guide
If the ACL is torn, it is replaced by graft. First step presents removal of the torn ACL. After that, part of patella, its tendon and a piece of tibial bone are taken out to make ACL graft. Next, tibial guide (Figure 1b) is placed onto tibial bone to help position the drill at the proper angle and a drill hole and the tunnel through tibia are created. Then, tunnel in the femur is created via previously created tibia tunnel by use of femoral guide (Figure 1c). Graft is then pulled out through created holes and secured by screws. If restorations of ACL and bone tunnels are more anatomically placed, knee stability and kinematics are better. Also, ACL attachment on tibial bone is surrounded by important anatomic structures, such as meniscal and cartilage areas and it is very important that in the reconstruction of the ACL these structures remain undamaged.

Tibial and femoral ACL attachment sites can vary in size. Average measured tibial ACL insertion site area measured at 46 patients according to [2] was $114 \mathrm{~mm}^{2}$, and can vary from $67-259 \mathrm{~mm}^{2}$. Luites et al. [3] measured this area at 35 cadaveric knees and it was $229 \pm 53 \mathrm{~mm}^{2}$. In a study [2] average measured femoral ACL insertion site measured at 50 patients was $83 \mathrm{~mm}^{2}$ and can vary from 46-156 $\mathrm{mm}^{2}$. As can be seen, ACL attachment sites areas can differ significantly for every patient and pre-operative planning procedure for ACL reconstruction for every patient can lead to more anatomically placed restoration of ACL. In paper [4] tibial bone model is generated for 10 patients from DICOM (Digital Imaging and Communication in Medicine) series of images and by the developed program system, analysis for different tibial guide parameters is presented. It is shown that for the

\footnotetext{
*Correspondence Author's Address: University of Novi Sad, Faculty of Technical Sciences, Trg Dositeja Obradovica 6, 
same tibial guide parameters, generated tibial aperture differs for all patients. It is concluded that a complex tibial bone anterior cruciate ligament insertion site geometry is different for every patient, so it is required that in the anterior cruciate ligament reconstruction analysis 3D tibial bone geometry should be taken into consideration. This can lead to the more precise native anterior cruciate ligament reconstruction.

In this paper, a methodology for knee bones models 3D printing from DICOM CT (Computed Tomography) series of images is presented.

From a series of images, tibial and femoral bones models are generated first. Next, from femoral bone points cloud, rotational axis (femoral transverse axis) about witch tibial bone rotates around femoral bone (knee flexion and extension) is calculated. After that, bone models and axis joint are prepared for 3D printing. Because of complex geometry, bones are printed as two parts on MakerBot Replicator 2X 3D printer and after that they are glued together. Model generated in this way could be very useful for preoperative planning. Before the reconstruction process, doctors would be able to simulate all parameters of reconstruction on printed 3D bones.

In the following chapters, details and results of developed methodology are presented.

\section{PROCEDURE OF 3D PRINTING KNEE BONES MODELS}

Procedure of printing knee bones models from DICOM series of images consists of three steps (Figure 2). First step generates models of tibial and femoral bones from DICOM series of images. Then, bone models are exported in STL (STereo Lithography) file format which can store bone geometry in polygonal format.

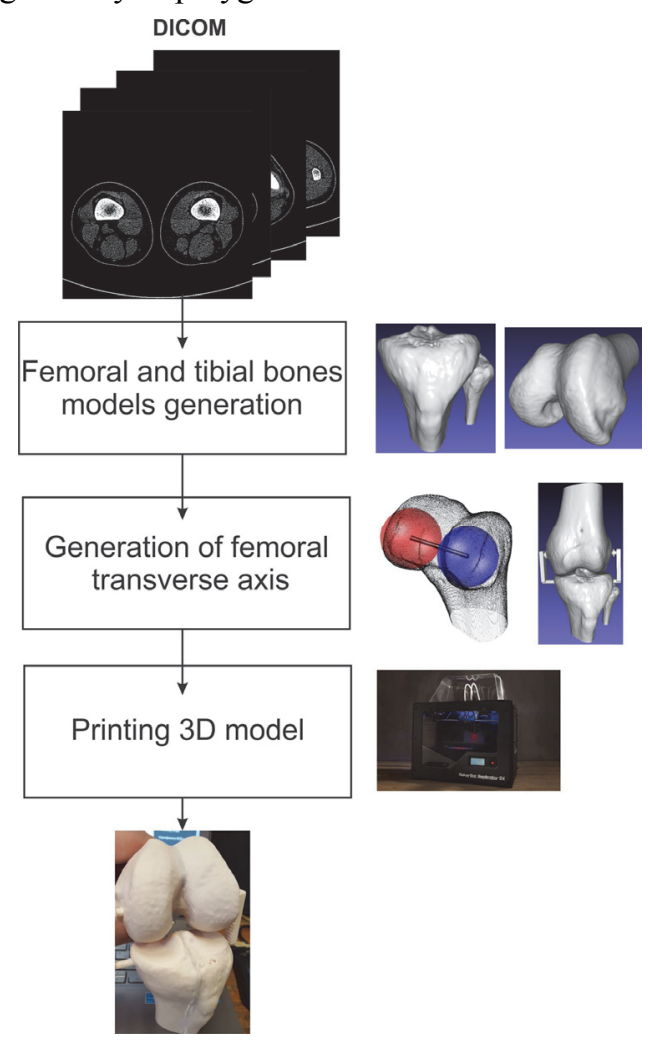

Fig.2. Steps in 3D printing knee bones models from DICOM series of images
Second step is generation of femoral transverse axis based on the femoral bone medial and lateral condyles point clouds. Tibial bone rotates about this axis around femoral bone in the ACL reconstruction procedure. Third step presents a $3 \mathrm{D}$ printing of generated bones models on MakerBot Replicator 2X printer.

In the next chapters these steps are presented in more details.

\subsection{Bone Models Generation from DICOM}

For the purpose of bones models generation, a program which uses VTK (Visualization ToolKit) open source library [5] is developed. It is a $\mathrm{C}++$ library and enables DICOM format reading, applying different filters on CT images, mesh generation and file export. In Figure 3, procedure for tibial and femoral bones models generation is presented.

In modern medical diagnostics, archivation of images is performed by using DICOM format records, covered by ISO 12052. Images are generated on CT (Computed Tomography) scanner.

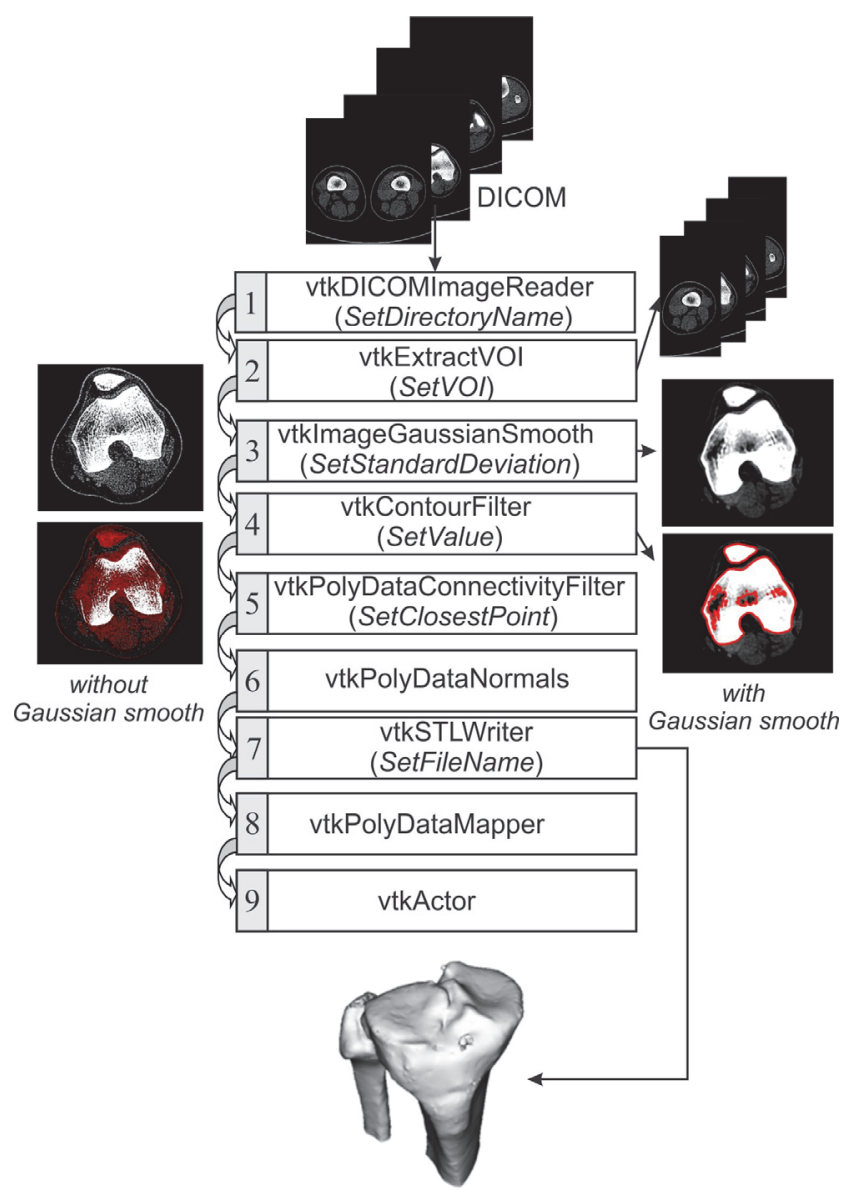

Bone model STL file

Fig.3. Procedure for tibial and femoral bones models generation by VTK library

Series of images in DICOM format present input in this system. First step presents reading raw scanned data in DICOM format by vtkDICOMImageReader class. On every image both legs are scanned. For pre-operative planning only knee with tear ACL is important, because ACL reconstruction process is to be performed on it. Extraction of part of an image which consists of knee with 
teared ACL can be done by vtkExtractVOI class. For model generation in VTK library vtkContourFilter class is responsible. Depending on input, this class generates 2D contours (if input is only one image), or a $3 \mathrm{D}$ polygonal model (if input is defined as a series of $2 \mathrm{D}$ images) for a defined isovalue parameter. Isovalue parameter is a float number which is saved at any pixel on the image and every tissue has a different value. Depending on the CT parameters, there can be a noise in the pictures. This noise can cause that final generated knee bones are connected together and in our case where bones should be separate to obtain their movement that could present a problem.or the noise reduction in the developed program system vtkImageGaussianSmooth class is used. Result of the 2D contouring on one image without noise reduction are shown on Figure 3 left, and with noise reduction on Figure 3 right. Generated contours are shown in red.

Generation of separate bone models (tibial and femoral bones) can be done by vtkPolyDataConnectivityFilter class. User should select point on a model by SetClosestPoint function and 3D model mesh which is closest to this point will get selected. Generation of model normals can be done by vtkPolyDataNormals class. Next step writes STL file of 3D bone model, by vtkStlWriter class. Polygonal 3D model geometry is mapped by vtkPolyDataMapper and for geometry display vtkActor class is used. In Figure 3 bottom, example of generated tibial bone model is shown.

\subsection{Calculation of Femoral Transverse Axis}

In the ACL reconstruction procedure, femoral bone is fixed and tibial bone can rotate (flexes and extends). One of the important factors is femoral transverse axis about which tibial bone rotates around femoral bone. According to [6] femoral transverse axis connects centers of the best fit spheres to the medial and lateral femoral bone condyles (Figure 4b). According to [7] femoral transverse axis passes through the center points of the best fit circles of the femoral bone lateral and medial condyles. In this paper authors used first approach with spheres, because 3D femoral bone model is generated in the step above by VTK library.

First, from femoral bone STL model, points cloud is generated (Figure 4a). After that only points on both condyles are saved (Figure $4 \mathrm{~b}$ ). To best fit spheres, for both condyles point clouds, the least square method is applied to the sphere equation similar as in [8]:

$$
\sum_{i=1}^{n} e^{2}=\sum_{i=1}^{n}\left\{\sqrt{\left(x_{i}-x_{c}\right)^{2}+\left(y_{i}-y_{c}\right)^{2}+\left(z_{i}-z_{c}\right)^{2}}-r\right\}^{2}
$$

In this case, the unknown $r, x_{\mathrm{c}}, y_{\mathrm{c}}$ and $z_{\mathrm{c}}$ values are calculated from a system of four equations that are obtained by setting the first partial derivative of equation (1) for all variables to zero. For this calculation MATHLAB 2010 is used.

Calculated centres of both best fit spheres lay on the femoral transverse axis. To enable that tibial bone can rotate about this axis, axis is modelled and added to the femoral and tibial bones (Figure 4d).

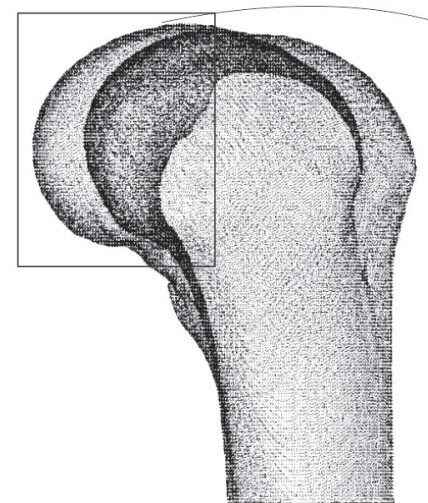

a)

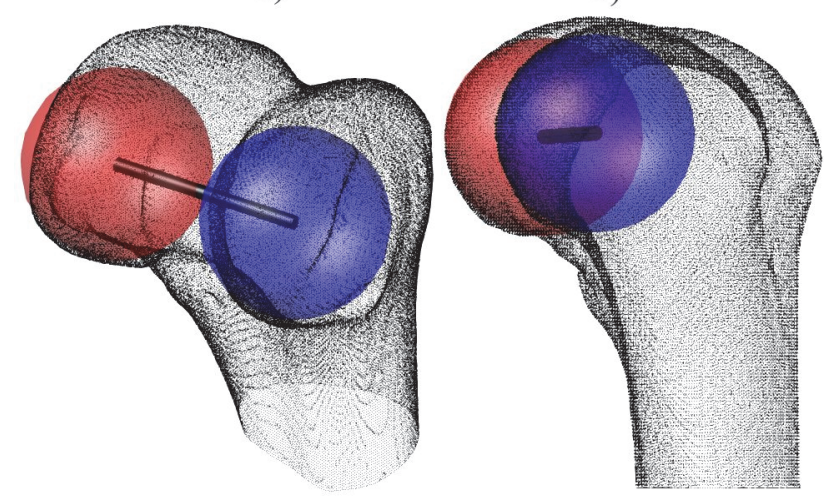

c)

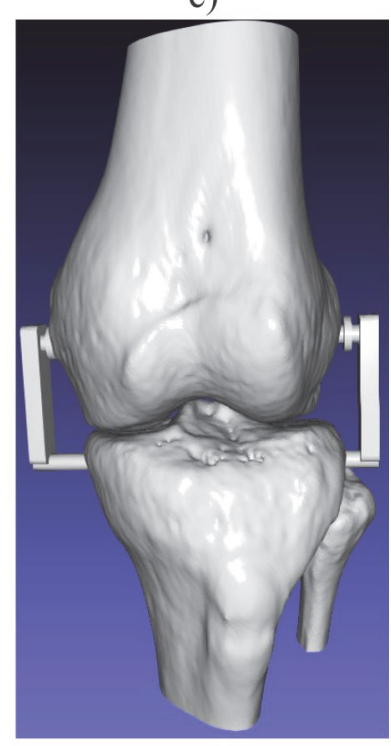

d)

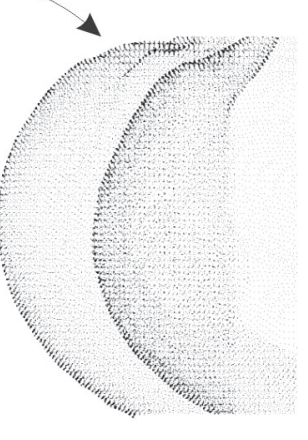

b)

.



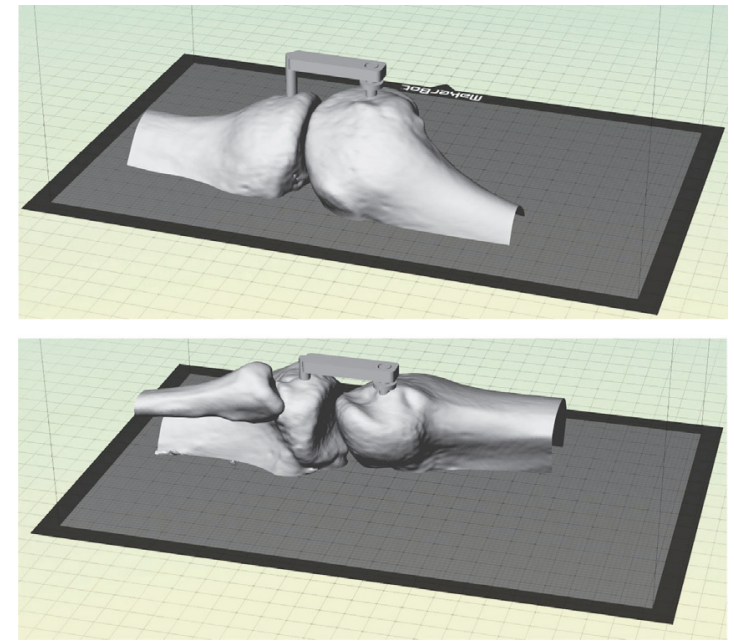

Fig.5. Model position in the printing process

Printing time for both parts of the model was approximately 12 hours. Both parts of the model are glued with a mixture of $\mathrm{ABS}$ and acetone. In Figure 6, final printed model is presented.
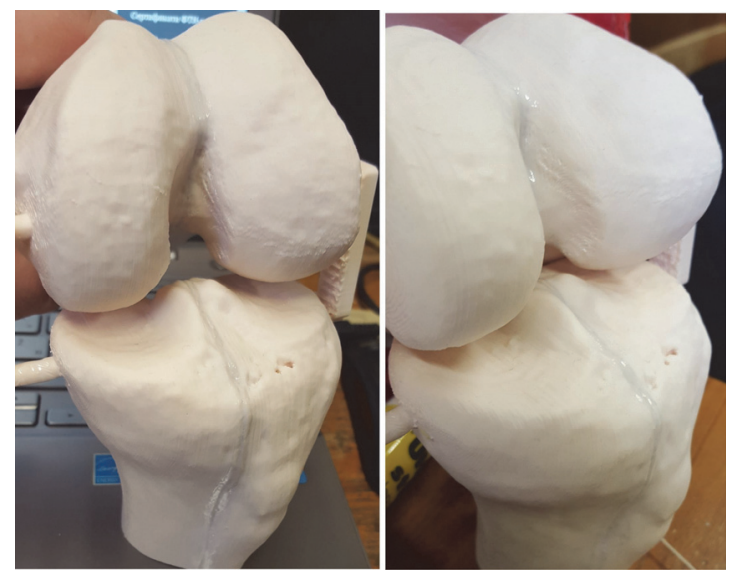

Fig.6. Printed model of femoral and tibial bone with femoral transverse axis

\section{CONCLUSION}

In this paper a methodology for knee bone models 3D printing from DICOM CT series of images is presented. Methodology consists of three steps. First step presents generation of tibial and femoral bone STL models generation. In the second step femoral transverse axis is calculated from centres of best fit spheres of femoral condyles points clouds. Also, this axis is modelled and added to the bones models. Last step presents printing models on $3 \mathrm{D}$ printer. In the ACL reconstruction, main goal is in positioning tibial and femoral drill guides more precisely to native position of ACL. With methodology presented in this paper, 3D printed knee bones could enable doctors to check all parameters before the actual ACL reconstruction. This could lead to the more precise native anterior cruciate ligament reconstruction.

\section{ACKNOWLEDGEMENTS}

In this paper some results of the project: Contemporary approaches to the development of special solutions related to bearing supports in mechanical engineering and medical prosthetics - TR 35025, carried out by the Faculty of Technical Sciences, University of Novi Sad, Serbia, are presented. Supported by the Ministry of Education, Science and Technological Development of Republic of Serbia.

\section{REFERENCES}

[1] Kopf, S., Martin, D.E., Tashman, S., Fu, F.H. (2010). Effect of tibial drill angles on bone tunnel aperture during anterior cruciate ligament reconstruction. $J$ Bone Joint Surg Am, 92 (4):871-881.

[2] Siebold, R., Ellert, T., Metz, S., Metz, J. (2008). Tibial insertions of the anteromedial and posterolateral bundles of the anterior cruciate ligament: morphometry, arthroscopic landmarks, and orientation model for bone tunnel placement. Arthroscopy, 24 (2):154-161.

[3] Luites, J.W., Wymenga, A.B., Blankevoort, L., Kooloos, J.G. (2007). Description of the attachment geometry of the anteromedial and posterolateral bundles of the ACL from arthroscopic perspective for anatomical tunnel placement. Knee Surg Sport Tr A, 15 (12):1422-1431.

[4] Milojević, Z., Tabaković, S., Vićević, M., Obradović, M., Vranješ, M., Milankov, M.Ž. (2016). The tibial aperture surface analysis in anterior cruciate ligament reconstruction process. Medicinski pregled, 69 (34):99-105.

[5] Schroeder, W.J., Lorensen, B., Martin, K. (2004). The visualization toolkit: an object-oriented approach to $3 D$ graphics, Kitware.

[6] Victor, J. (2009). Rotational alignment of the distal femur: a literature review. Orthopaedics \& Traumatology: Surgery \& Research, 95 (5):365-372.

[7] Howell, S.M., Howell, S.J., Hull, M.L. (2010). Assessment of the radii of the medial and lateral femoral condyles in varus and valgus knees with osteoarthritis, JBJS, 92 (1):98-104.

[8] Tabakovic, S., Zeljkovic, M., Milojevic, Z. (2014). Automated Acquisition of Proximal Femur Morphological Characteristics. Measurement Science Review, 14 (5):285-293.

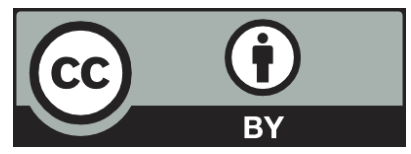

(C) 2017 Authors. Published by the University of Novi Sad, Faculty of Technical Sciences.

This article is an open access article distributed under the terms and conditions of the Creative Commons Attribution license 3.0 Serbia (http://creativecommons.org/licenses/by/3.0/rs/). 